\section{Intracerebral hemorrhage associated with vaccine-induced thrombotic thrombocytopenia following ChAdOx1 nCOVID-19 vaccine in a pregnant woman}

Severe acute respiratory syndrome coronavirus 2 (SARS-CoV-2) spread globally, causing significant morbidity and mortality. Therefore, highly effective vaccines play an important role in increasing population immunity and preventing severe disease. The ChAdOx1 nCoV-19 (Oxford-AstraZeneca) vaccine consists of a replicationdeficient chimpanzee adenoviral vector containing the SARS-CoV-2 structural surface glycoprotein spike antigen gene. ${ }^{1}$ No signal for increased thrombotic events was detected in clinical trials of this vaccine involving 23,848 participants, ${ }^{1}$ which has now been administered to more than 34 million people worldwide. ${ }^{2}$ As reported to date, 20.8 million doses of the ChAdOx $1 \mathrm{nCoV}-19$ vaccine have been applied in Brazil. ${ }^{3} \mathrm{ChAdOx} \mathrm{nCoV}-19$ has been associated with vaccine-induced thrombotic thrombocytopenia (VITT), ${ }^{4}$ a rare but serious adverse event. The potential underlying associated mechanism is the formation of anti-heparin/platelet factor 4 (PF4) antibodies, but risk factors other than young age remain unclear. ${ }^{2,5}$ The immune-driven disease resembles heparin-induced thrombocytopenia (HIT) because platelet-activating antibodies recognize multimolecular complexes between cationic PF4 and anionic heparin, but classically there is no heparin exposure in VITT. ${ }^{5}$ So far, the incidence of VITT is around one case per 100,000 exposures to the ChAdOx1 nCoV-19 vaccine. ${ }^{2}$ Clinically, it is characterized by thrombocytopenia and thrombosis in unusual sites, particularly cerebral venous or splanchnic-vein thrombosis. Cerebral venous sinus thrombosis (CVST), a life-threatening event, was described in $72 \%$ of the VITT reports. ${ }^{6}$ Clinical trials did not include pregnant women, and the available data came from accidental pregnancies. Pregnant patients with COVID-19 are more likely to die or need intensive care compared with non-pregnant persons of reproductive age. ${ }^{1}$ Herein we present a fatal case of a 35-year-old pregnant woman who developed intracerebral hemorrhage in the left temporal lobe associated with VITT 12 days after the off-label ChAdOx1 nCOVID-19 vaccination and we show the characterization of the hemostatic profile. A 35-years-old white pregnant woman at 23 weeks gestation was in prenatal follow-up with normal platelet counts and controlled hypothyroidism due to Hashimoto's disease. She received the first dose of the ChAdOx1 nCov-19 vaccine in late April 2021 (day 0). The next day, she reported having minor symptoms (malaise, chills, tremors, and "cold feeling"). On day 2, she presented a generalized skin rash on her legs, abdomen, and back with spontaneous resolution (see Figure 1A). Prenatal exams on day 3 identified urinary tract infection caused by Morganella morganii treated with trimethoprim-sulfamethoxazole and $148,000 / \mathrm{mm}^{3}$ platelets at the routine complete blood count. On day 7 , she presented headache, nausea, nonspecific malaise, polaciuria, pain in the lower limbs and hips. Despite having $121,000 / \mathrm{mm}^{3}$ platelets, she received analgesics and was discharged from the obstetric emergency room. On the following days, she maintained headache and bilateral leg pain, partially responsive to dipyrone. On day 11 , she reported a severe headache located in the left maxillary region, and on day 12 , she was admitted with $33,000 / \mathrm{mm}^{3}$ platelets and an excruciating headache. During the computed tomography (CT) exam, she became comatose and underwent endotracheal intubation, which precluded the realization of CT cerebral venography, confirming the suspicion of central venous sinus thrombosis. Brain CT showed a large acute intraparenchymal hematoma in the temporal lobe, insula, and temporoparietal transition of the left cerebral hemisphere measuring approximately $10.1 \times 5.4 \times 5.5 \mathrm{~cm}$. The hematoma was surrounded by vasogenic edema with midline shift to the right, herniation of the uncus, marked compressive effect on the midbrain, and subtotal collapse of the supratentorial ventricular system (Figure 1B). The patient received platelet concentrate transfusion and underwent urgent neurosurgery for hematoma drainage and decompressive craniectomy. After receiving critical care procedures, obstetric ultrasound detected fetal death. There were no platelet clumps, signs of erythrocyte fragmentation, or blast cells on blood film. She

Table 1. Laboratory characteristics of the patient.

\begin{tabular}{|c|c|c|c|}
\hline Laboratory analysis & Reference value & D12 & D15* \\
\hline Hemoglobin $(\mathrm{g} / \mathrm{dL})$ & $10.5-14.8$ & 12.7 & 6.2 \\
\hline Platelet count (per mm³) & $155,000-409,000$ & 33,000 & 32,000 \\
\hline Leucocytes $($ per mm³) & $4,500-11,000$ & 12,199 & 10,030 \\
\hline D-dimer (ng/mL) & $298-1,653$ & $>20,000$ & - \\
\hline Fibrinogen (mg/dL) & $340-853$ & 221 & - \\
\hline Activated partial thromboplastin time (rel) & $<1.25$ & 1.04 & - \\
\hline International normalized ratio & $0.8-1.2$ & 1.17 & - \\
\hline Lactate dehydrogenase (UI/L) & $81-234$ & 164 & - \\
\hline C-reactive protein (mg/dl) & $<0.3$ & 6.00 & 16.54 \\
\hline Aspartate aminotransferase (U/L) & $15-37$ & 57 & - \\
\hline Alanine aminotransferase (U/L) & $6-45$ & 37 & - \\
\hline Thyroid stimulating hormone $(\mu \mathrm{UI} / \mathrm{mL})$ & $0.4-4.3$ & - & 10.03 \\
\hline Free thyroxine (ng/dL) & $0.93-1.7$ & - & 0.99 \\
\hline Anti-heparin/ PF4 ELISA (OD) & $\leq 0.4$ & $>10$ & - \\
\hline IL18 (pg/mL) & $315 \pm 36$ & - & 1,075 \\
\hline $\operatorname{IL} 1 \beta(\mathrm{pg} / \mathrm{mL})$ & $4 \pm 8$ & - & 92 \\
\hline
\end{tabular}

*Functional assay. OD: optical density; ELISA: enzyme-linked immunosorbant assay. IL: interleukin: D: day. 
A

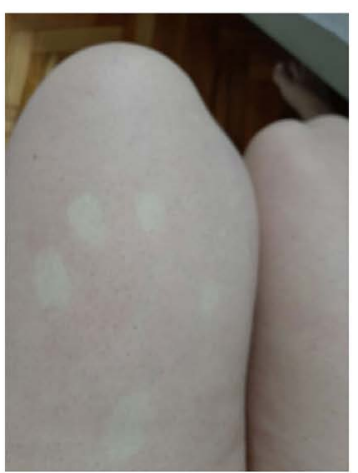

D

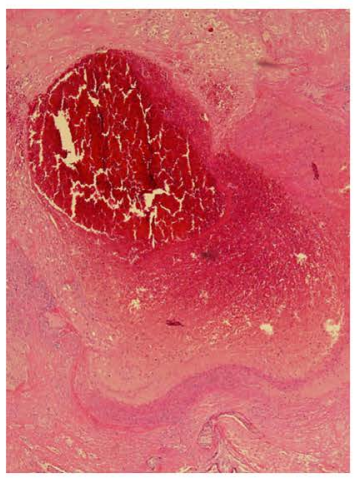

B

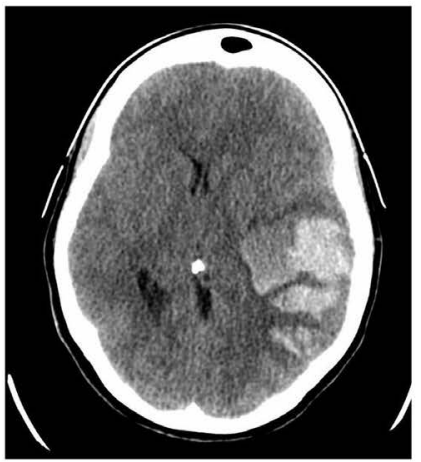

E

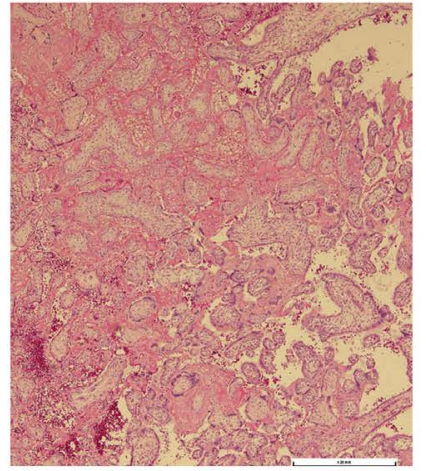

C

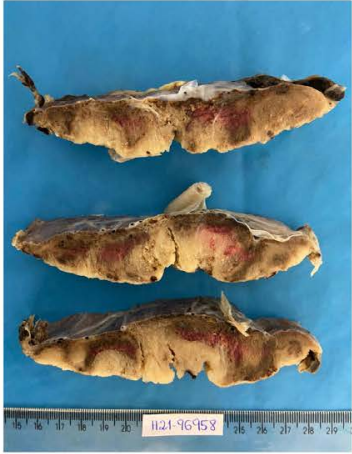

$\mathbf{F}$

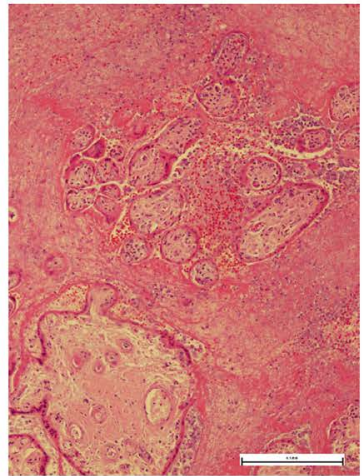

Figure 1. Pictures of skin rash, cerebral hemorrhage, and placenta. (A) Generalized skin rash on legs with spontaneous resolution; (B) brain computed tomography with large acute intraparenchymal hematoma in the temporal lobe, insula, and temporoparietal transition of the left cerebral hemisphere measuring approximately $10.1 \times 5.4 \times 5.5 \mathrm{~cm}$ surrounded by vasogenic edema with midline shift to the right, herniation of the uncus, marked compressive effect on the midbrain and subtotal collapse of the supratentorial ventricular system; (C) cut surface of the placenta shows pale parenchyma with irregular and firm red-brown areas; (D) occlusive thrombus in a decidual vessel surrounded by a recent infarct; (E) chorionic villi encased by perivillous fibrin and red blood cells with some preservation of the trophoblast layer and villous stroma; villous capillaries are sparse; (F) area with massive intervillous fibrin deposition, some leukocytes and red blood cells with degenerative villous configuring an intervillous thrombosis.

received $80 \mathrm{~g}(1 \mathrm{~g} / \mathrm{Kg})$ immunoglobulin $(\mathrm{Ig})$ and $2 \mathrm{~g}$ fibrinogen without success. The patient died on day 17 (after vaccination) with refractory intracranial hypertension despite all pressure control measures. SARS-CoV-2 reverse transcription polymerase chain reaction (RT-PCR) of nasopharyngeal swab and placenta was negative. Serology to dengue, Chikungunya, Zika, HIV, hepatitis B and $\mathrm{C}$, cytomeglaovirus, Eppstein-Barr virus, toxoplasmosis, and rubella were negative. Relatives denied past COVID-19 infection, heparin exposure, or hormone therapy. There was no personal or familial history of thrombosis or miscarriage. Laboratory results are depicted in Table 1. The d-dimer level was over $20.000 \mathrm{ng} / \mathrm{dL}$ and fibrinogen $221 \mathrm{mg} / \mathrm{dL}$ (reference in the second trimester of pregnancy $298-1,653 \mathrm{ng} / \mathrm{mL}$ and $340-853 \mathrm{mg} / \mathrm{dL}$, respectively). The results of other blood tests were normal except for alanine aminotransferase and C-reactive protein levels, which were increased. Levels of the proinflammatory cytokines IL-1 $92 \mathrm{pg} / \mathrm{mL}$ and IL-18 1,075 $\mathrm{pg} / \mathrm{ml}$ were increased (compared to healthy controls at $4 \pm \beta 8 \mathrm{pg} / \mathrm{mL}$ and $315 \pm 36 \mathrm{pg} / \mathrm{mL}$, respectively). Screening for hereditary thrombophilia with factor $V$ Leiden and prothrombin 20210 mutation test was negative. Antinuclear antibodies, anticardiolipin IgG and IgM, lupus anticoagulant, and $\beta 2$ glycoprotein $1 \mathrm{IgG}$ antibodies were not detected. There were no signs of hemolysis. Anti-heparin/PF4 enzyme-linked immunosorant assay (ELISA) IgG antibodies were detected with an optical density value above 10 (reference $\leq 0.4$ ). The pathological study of the placenta revealed intense inflammatory activity with increased intervillous fibrin-thrombotic areas, villous collapse configuring preinfarcted areas, and recent infarctions and focuses of decidual vessel throm- bosis (Figure $1 \mathrm{C}$ to $\mathrm{F}$ ). Platelet activation and functional assay were performed as described. ${ }^{9,10}$ As shown in Figure 2, platelets from the patient exhibited signs of activation. Increased CD63 surface expression, a marker of dense granules secretion, was observed when compared to control (Figure 2A and B). Moreover, although platelet surface expression of CD62 $p$ was not different between the patient and controls, elevated levels of circulating sCD62 $p$ and platelet-derived microparticles expressing CD62 $p$ were observed in the patient compared to controls (Figure 2C to E). Functional activity of patient plasma to activate platelets from healthy volunteers was demonstrated in Figure $2 \mathrm{~F}$ to $\mathrm{H}$. Patient plasma trigger increased surface expression of CD62p, CD63, and the release of platelet-derived microparticles expressing CD62p. Conversely, plasma-induced platelet activation was inhibited by a high concentration of heparin (100 $\mathrm{IU} / \mathrm{mL}$ ). We present the first report of VITT following ChAdOx1 nCOVID-19 vaccination in a pregnant woman. Despite the lack of image verification of cerebral thrombosis, clinical features were suggestive. Initial brain CT may be negative for CVST in about $30 \%$ of cases. ${ }^{11}$ Besides that, thrombosis was observed in placental vessels. The constellation of signs and symptoms suggests VITT diagnosis complicated by intracranial hemorrhage with a fatal outcome both to fetus and mother. Three independent descriptions of 39 persons presenting VITT after vaccination with ChAdOx1 $\mathrm{nCoV}-19$ revealed a vast majority being women younger than 50 years of age and some of them receiving estrogen-replacement therapy or oral contraceptives. Nevertheless, most of the participants did not have preexisting risk factors for thrombosis. ${ }^{2}$ Patients presenting VITT have unusually severe 
A

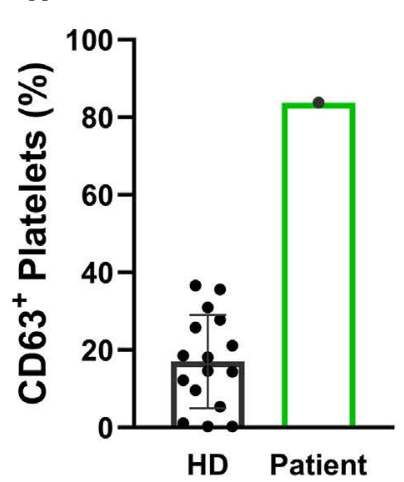

C

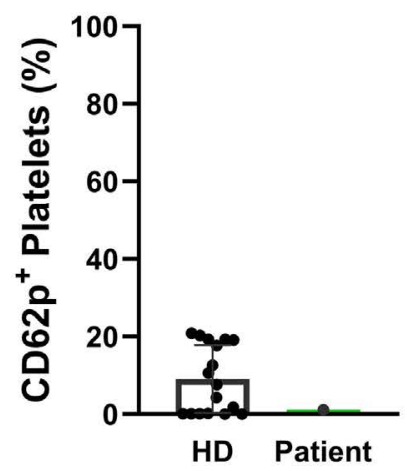

F

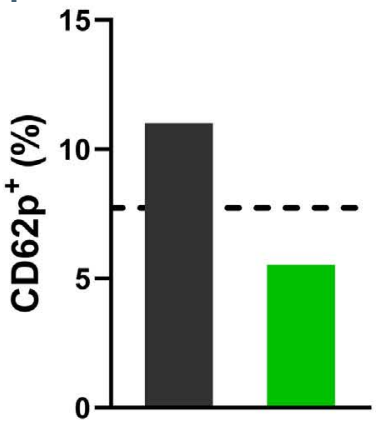

B

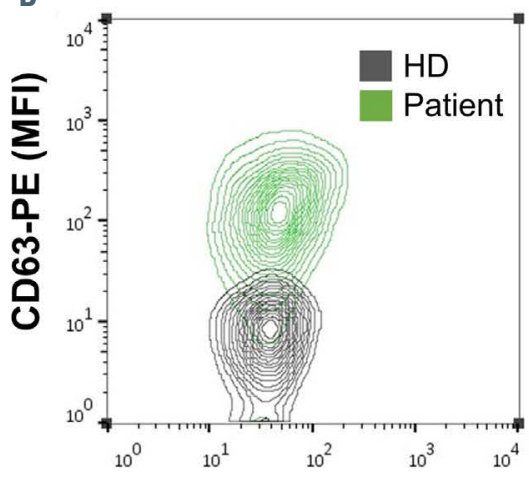

Side Scatter

D

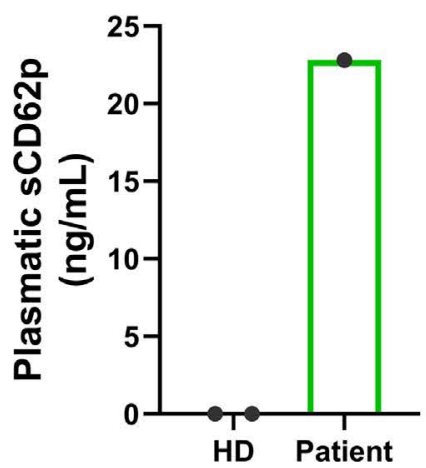

E

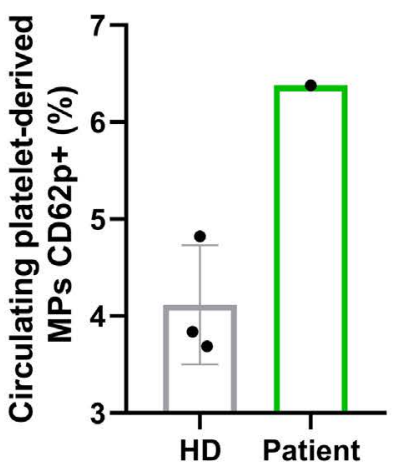

Patient's plasma

Patient's plasma + Heparin $(100 \mathrm{UI} / \mathrm{mL})$
G

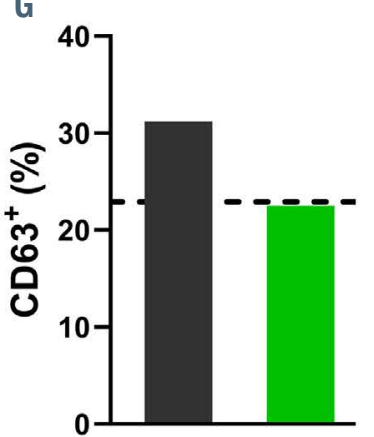

H

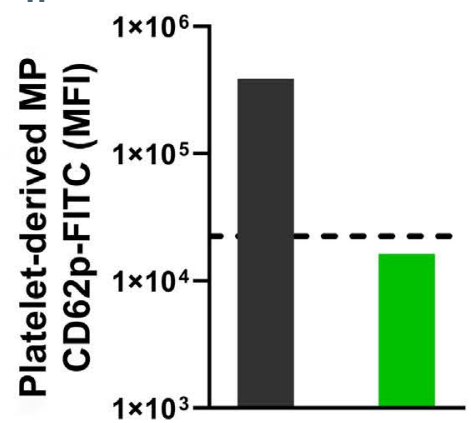

Figure 2. Increased platelet activation and plasma-induced platelet activation. The percentage of platelets expressing CD63 (A) and CD62p (C) are shown on platelets isolated from healthy donors (HD) or the patient. Representative contour plot of CD63 expression (mean fluorescence intensity, MFI) by side scatter of $\mathrm{HD}$ (grey) or patient (green) platelets (B). The concentration of soluble P-selectin (SCD62P) in the plasma from HD or the patient (D). The percentage of circulating platelet-derived microparticles (MP) expressing CD62p (E). The percentage of platelets expressing CD62p (F) and CD63 (G) after incubation with the patient's plasma (10\%) with (green) or without (grey) the presence of heparin (100 UI/mL). The MFI of CD62p-FITC in platelet-derived microparticles from the supernatant of HD platelets stimulated with the patient's plasma with (green) or without (grey) the presence of heparin $(100 \mathrm{UI} / \mathrm{mL})(\mathrm{H})$. The dotted lines represent the values of the control groups (HD platelets incubated with heterologous HD plasma).

thrombocytopenia, increased frequency of disseminated intravascular coagulation, and atypical thrombotic events. Serum from these patients shows a strong reactivity on the PF4-heparin ELISA and activates platelets in the presence and absence of heparin, but a high concentration of heparin completely inhibits the effect, ${ }^{5}$ as occurred in our case. In addition, we observed platelet activation confirmed by increased CD63 surface expression, circulating sCD62 $\mathrm{p}$ levels, and platelet-derived microparticles expressing CD62p. Interestingly, we identified an elevation in the patient's plasma of IL-1 $\beta$ and IL-18 compared to controls. Of note, IL-1 $\beta$ and IL-18 are central mediators of inflammation and thrombosis, released upon inflammasome activation. ${ }^{12}$ Indeed, inflammasome activation and IL- $1 \beta$ release play multiple functions that may favor vein and arterial thrombosis including the induction of procoagulant activity, the promotion of leukocyte adhesion to vascular endothelial cells, induction of neutrophil extracellular traps, and pyroptosis. ${ }^{13}$ Future studies should address whether or not this is also observed in other VITT cases and participate in the disease pathophysiology. Stroke affects 30 per 100,000 pregnancies, with ischemia, CVST, and hemorrhage causing roughly equal numbers and the highest risk in peripartum and postpartum. ${ }^{14}$ However, it is unclear if the pregnancy itself could increase the risk for thrombotic events following ChAdOx1 nCov-19 vaccination with the formation of anti-PF4 antibodies. Another element that should be pointed out, in this case, is the history of thyroiditis. Hypothyroidism is an underestimated risk 
factor for CVST, although most of patients have additional risk factors, suggesting a multifactorial hypercoagulability. ${ }^{11}$ This case report draws attention to this possible severe vaccine side effect among pregnant women and the challenges related to an early diagnosis. When symptomatic, pregnant women experience difficulties diagnosing VITT due to avoidance of screening methods involving radiation and presenting features possibly related to pregnancy, such as thrombocytopenia. The presence of persistent headache and thrombocytopenia within 30 days of vaccination should raise the suspicion of VITT. $\mathrm{D}$-dimer, fibrinogen, and imaging exams help to guide prompt treatment, preventing a rapidly progressive disease..$^{5}$ At least five countries had instituted limitations primarily based on age on which patients should receive the ChAdOx1 nCoV-19 vaccine, ${ }^{2}$ and Brazil recently temporarily suspended the administration of this vaccine in pregnant women. ${ }^{15}$ Those either affected by VITT or under investigation for this complication should not receive a second $\mathrm{ChAdO} \times 1 \mathrm{nCoV}-19$ vaccine. Physicians should have a low threshold for recognizing VITT signs and symptoms and requesting ELISA testing for PF4-polyanion antibodies and confirmatory functional tests. Although rare, VITT is a new phenomenon with devastating effects for otherwise healthy young adults, and its association with COVID-19 vaccination requires a thorough risk-benefit analysis especially for pregnant women.

Daniela P. Mendes-de-Almeida, ${ }^{1,2,3}$ Remy MartinsGonçalves, ${ }^{4}$ Renata Morato-Santos, ${ }^{5}$ Gustavo Adolpho C. de Carvalho, ${ }^{6,7}$ Silas A. Martins, ${ }^{6,7}$ Lohanna Palhinha, ${ }^{4}$ Vanessa Sandim, ${ }^{8}$ Elyzabeth Avvad-Portari, 9,10 Fernando A. Bozza, ${ }^{11,12}$ Robson Q. Monteiro, ${ }^{8}$ Patrícia T. Bozza $a^{4}$ and Pedro Kurtz ${ }^{12,13,14}$

1 Department of Hematology, Instituto Nacional de Infectologia Evandro Chagas, Fundação Oswaldo Cruz (FIOCRUZ), Rio de Janeiro, Brazil; 'Division of Molecular Carcinogenesis, Research Center, Instituto Nacional de Câncer (INCA), Rio de Janeiro, Brazil; ${ }^{3}$ Division of Epidemiology, Department of Pediatrics, University of Minnesota, Minneapolis, MNS, USA; ${ }^{4}$ Laboratory of Immunopharmacology, Instituto Oswaldo Cruz, Fundação Oswaldo Cruz (FIOCRUZ), Rio de Janeiro, Brazil; '5epartment of Obstetrics, Universidade Federal do Estado do Rio de Janeiro (UNIRIO), Rio de Janeiro, Brazil; ${ }^{6}$ Neurosurgical Deptartment, Hospital Sao Lucas, Rio de Janeiro, Brazil; ${ }^{7}$ Neurosurgical Deptartment, Hospital Adventista Silvestre, Rio de Janeiro, Brazil; ' Institute of Medical Biochemistry Leopoldo de Meis, Universidade Federal do Rio de Janeiro (UFRJ), Rio de Janeiro, Brazil; 'Department of Pathologic Anatomy, Instituto Nacional de Saúde da Mulher, da Criança e do Adolescente Fernandes Figueira, Fundação Oswaldo Cruz (FIOCRUZ), Rio de Janeiro, Brazil; ${ }^{10}$ Department of Pathologic Anatomy, Histology, and Embryology, Medical Sciences Faculty, Universidade do Estado do Rio de Janeiro (UERJ), Rio de Janeiro, Brazil; "Laboratory of Intensive Care, Instituto Nacional de Infectologia Evandro Chagas, Fundação Oswaldo Cruz (FIOCRUZ), Rio de Janeiro, Brazil; ${ }^{12} D$ 'Or Institute for Research and Education, Rio de Janeiro, Brazil; ${ }^{13}$ Department of Neurointensive Care, Instituto Estadual do Cérebro Paulo Niemeyer (IECPN), Rio de Janeiro, Brazil and ${ }^{14}$ Department of Intensive Care Medicine, Hospital Copa Star, Rio de Janeiro, Brazil

$$
\begin{aligned}
& \text { Correspondence: PEDRO KURTZ - kurtzpedro@mac.com } \\
& \text { doi:10.3324/haematol.2021.279407 } \\
& \text { Received: June 14, 2021. }
\end{aligned}
$$

\section{Accepted: July 8, 2021}

Pre-published: July 15, 2021.

Disclosures: no conflicts of interest to disclose.

Contributions: DPMA, PTB, and PK designed the study; DPMA, RMS, GACC, $S A M$, and $P K$ attended the patient and analyzed the clinical data; DPMA, RMG, and PTB wrote the manuscript. $R M G, L P$, and VS performed the functional assay; EAP performed the pathological examination; $R Q M, F A B$, and $P T B$ revised the manuscript and supervised the study. All authors saw and approved the manuscript and its submission.

Acknowledgments: we thank the patient's relatives for the informed consent, Dr. Beatriz Grinsztejn, Dr. Valdiléia Veloso, Dr. Maria de Lourdes Maia and Filipe Santos-Bueno for technical support, and Prof. Logan Spector for the language review.

Data sharing statement: the documentation is available on a reasonable request to the corresponding author.

\section{References}

1. Voysey M, Clemens SAC, Madhi SA, et al. Safety and efficacy of the ChAdOx1 nCoV-19 vaccine (AZD1222) against SARS-CoV-2: an interim analysis of four randomised controlled trials in Brazil, South Africa, and the UK. Lancet. 2021;397(10269):99-111.

2. Cines DB, Bussel JB. SARS-CoV-2 Vaccine-induced immune thrombotic thrombocytopenia. N Engl J Med. 2021;384(23):2254-2256.

3. Ministério da Saúde. Localiza SUS, Vacinômetro SUS. https://localizasus.saude.gov.br/. Accessed May 292021.

4. European Medicines Agency - Sciences Medicine Health. COVID19 Vaccine AstraZeneca: benefits still outweigh the risks despite possible link to rare blood clots with low blood platelets. https://www.ema.europa.eu/en/news/covid-19-vaccineastrazeneca-benefits-still-outweigh-risks-despite-possible-link-rareblood-clots. Accessed May 292021

5. Scully M, Singh D, Lown R, et al. Pathologic antibodies to platelet factor 4 after ChAdOx1 nCoV-19 vaccination. N Engl J Med 2021; 384(23):2202-2211.

6. See I, Su JR, Lale A, et al. US case reports of cerebral venous sinus thrombosis with thrombocytopenia after Ad26.COV2.S vaccination, March 2 to April 21, 2021. JAMA. 2021;325(24):2448-2456.

7. Male V. Are COVID-19 vaccines safe in pregnancy? Nat Rev Immunol. 2021;21(4):200-201.

8. Zambrano LD, Ellington S, Strid P, et al. Update: characteristics of symptomatic women of reproductive age with laboratory-confirmed SARS-CoV-2 infection by pregnancy status - United States, January 22-October 3, 2020. MMWR Morb Mortal Wkly Rep. 2020; 69(44):1641-1647

9. Michelson AD, Barnard MR, Krueger LA, Frelinger AL, Furman MI. Evaluation of platelet function by flow cytometry. Methods. 2000; 21(3):259-270.

10. Hottz ED, Azevedo-Quintanilha IG, Palhinha L, et al. Platelet activation and platelet-monocyte aggregate formation trigger tissue factor expression in patients with severe COVID-19. Blood. 2020; 136(11):1330-1341.

11. Idiculla PS, Gurala D, Palanisamy M, Vijayakumar R, Dhandapani S, Nagarajan E. Cerebral venous thrombosis: a comprehensive review. Eur Neurol. 2020;83(4):369-379

12. Henao-Mejia J, Elinav E, Strowig T, Flavell RA. Inflammasomes: far beyond inflammation. Nat Immunol. 2012;13(4):321-324.

13. Takahashi M. NLRP3 inflammasome as a key driver of vascular disease. Cardiovasc Res. 2021 Jan 23;cvab010. [Epub ahead of print]

14. Swartz RH, Cayley ML, Foley N, et al. The incidence of pregnancyrelated stroke: A systematic review and meta-analysis. Int J Stroke. 2017;12(7):687-697.

15. Fantinato FFST, Cruz LM. Nota Técnica No 627/2021CGPNI/DEIDT/SVS/MS. Nota técnica no. 627/2021 https://saude.rs.gov.br/upload/arquivos/202104/27181903-nota-tecnica-467-2021-cgpni-deidt-svs-ms.pdf. Acessed May 242021. 